\title{
An Optimal Bandwidth Resource Utilization Scheme in IEEE 802.16m Based
} Networks

\author{
Hanwu Wang ${ }^{1}$, Beibei Yang ${ }^{2}$ and Liang $\mathrm{An}^{3}$ \\ ${ }^{123}$ College of Computer Science and Electronic Engineering, Hunan University, Changsha, Hunan, \\ China
}

Keywords: radio frequency; channel bandwidth; utilization optimization

\begin{abstract}
The IEEE $802.16 \mathrm{~m}$ based broadband wireless access is a brand-new technology to provide high speed and wide range communications for wireless users. However, how to exploit the optimal utilization of the radio frequency bands resource in such networks is a big challenging issue as it covers the radio channel capacity optimization, channel bandwidth utilization optimization and utilization performance analysis. In this regarding, we first propose a novel scheme to exploit the optimal channel transmission capacity based on the time varying state of each radio channel. Then we design an effective channel usage scheme to fully utilize the channel transmission bandwidth with respect to user's requirements. Next we also present a scheme to make the performance analysis on the proposed channel utilization scheme with respect to various metrics. Experiment results validate the efficiency and efficacy of the proposed schemes.
\end{abstract}

\section{Introduction}

Among all those IEEE 802.16 technologies, the 802.16m[1][2][3]defines a new mobile air interface between the base station(BS) and end mobile station(MS). The $802.16 \mathrm{~m}$ is based on the 802.16 single hop legacy but extends it to support the mobile multi-hop relay operation by introducing the low cost relay station(RS) into the network. The key purpose of an RS is to aid the good communication between BS and end MSs so that a better transmission speed and range can be achieved. Due to its significant merits, such as high transmission rate, wide coverage, mobility and flexibility, the IEEE $802.16 \mathrm{~m}$ is regarded as a promising alternative for providing broadband wireless access(BWA) in wide area communication field.

In an IEEE $802.16 \mathrm{~m}$ based BWA communication network, the key challenge is how to utilize the radio frequency resource and provide desired transmission resource (i.e.,channel transmission bandwidth) for each user to meet their individual service requirements. Accordingly, we propose novel schemes to adapt the state variation of radio communication channels and maximize the channel transmission rate and system capacity. Based on the obtained channel transmission bandwidth, we further propose an effective channel usage scheme to fully utilize bandwidth resource with respect to various user requirements. Moreover, we also construct an analytical model to make performance analysis for the proposed schemes.

\section{Optimization on Radio Channel Capacity}

As the channel state in a BWA communication network may vary from time to time, hence how to adapt the channel state variation and exploit the maximal channel transmission capacity is a critical challenge. The IEEE 802.16 standard has specified different modulation levels (types), such as BPSK, QPSK, 16-QAM, 64-QAM, etc[4][5] for data transmission according to the channel states, and each modulation type corresponds to one type of transmission rate. As is known, channel state captured by its SNR $r(t)$ is a time-variant quantity, hence we need to partition all those SNR values into different disjoint intervals and let each SNR interval represent a specific channel state. Let $0=r_{0}<r_{1}<r_{2}<\ldots<r_{\mathrm{N}}$ be the $N+1$ SNR thresholds; $\left[\mathrm{r}_{0}, \mathrm{r}_{1}\right),\left[\mathrm{r}_{1}, \mathrm{r}_{2}\right), \ldots,\left[\mathrm{r}_{\mathrm{N}-1}, \mathrm{r}_{\mathrm{N}}\right)$ be the $\mathrm{N}$ SNR intervals. A transmission channel is said to be in state $n(n=1,2, \ldots, N)$ if its SNR $r(t)$ lies within the interval $\left[r_{\mathrm{n}-1}, r_{\mathrm{n}}\right)$. Accordingly a SNR interval is matched with a modulation level and channel transmission rate. 


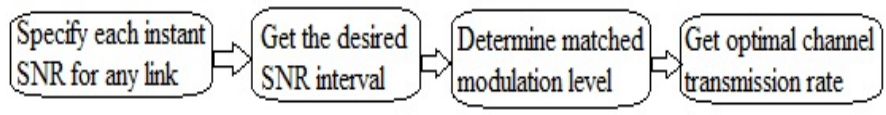

Fig. 1. Radio Channel Transmission Adaptation

The channel transmission optimization is described as in Figure 1 , where $\{[n-1, n)\}$ and $\left\{M L_{n}\right\}$ denotes the set of all possible SNR intervals and modulation levels respectively. As an IEEE 802.16 frame $\mathrm{f}$ is composed of $N$ time slots, $\mathrm{t}_{1}, \mathrm{t}_{2}, \ldots, \mathrm{t}_{\mathrm{N}}$, the channel transmission optimization works as follows: over each time slot $t_{\mathrm{k}}(k=1,2, \ldots, N)$, we first determines its average SNR for an arbitrary link $l$, then we can calculate crossing rate for each SNR level, i.e., $N_{\mathrm{n}}\left(r\left(t_{\mathrm{k}}\right)\right)$ as [6]

$$
N_{n}\left(r\left(t_{k}\right)\right)=\frac{\sqrt{2 \pi} f_{D}\left(t_{k}\right)}{\Gamma(m)}\left(\frac{m r_{n}}{r\left(t_{k}\right)}\right)^{m-1 / 2} \exp \left\{-\frac{m r_{n}}{r\left(t_{k}\right)}\right\}
$$

Among all those calculated $N_{\mathrm{n}}\left(r\left(t_{\mathrm{k}}\right)\right)$ values, we choose the maximal one and determine its relevant interval say $\left[n^{*}-1, n^{*}\right)$, and also get the matched modulation level $M L_{\mathrm{n}^{*}}$ and transmission rate $r\left(t_{\mathrm{k}}\right)$ for link $l$. As for the whole frame $\mathrm{f}$, the channel SNR of link $l$ over each time slot $t_{\mathrm{k}}(k=1,2, \ldots, N)$ may vary from one to another, hence one important task is how to partition all those time slots within $f$ into different so called bursts. Specifically a burst denotes a subset of continuous time slots where the channel state of link $l$ over those slots is the same (i.e.,belongs to the same SNR interval).

We construct a new burst by adding each appropriate slot into it in a sequence manner. As for each new burst $B_{\mathrm{i}}$, we first determine the desired channel state say $n^{*}$ for its first time slot $\mathrm{t}_{\mathrm{s}}$, then similarly we work on the next time slot $t_{\mathrm{e}}$ and get its SNR state. If two states are the same, then we can add te into burst $B_{\mathrm{i}}$ and further handle the remaining time slots; otherwise we let $t_{\mathrm{e}}$ be the first time slot of another new burst. For each round we can work out one burst with a definite channel state and its matched transmission rate $r\left(l, B_{\mathrm{i}}\right)$. In this way we can readily get all possible transmission bursts within $f$ for link $l$. Regarding all those bursts, the total transmission rate (capacity) of link $l$ within the current frame $f$ is calculated as $\left(\left|B_{\mathrm{i}}\right|\right.$ denotes the cardinality of $\left.B_{\mathrm{i}}\right)$

$$
R(f)=\sum_{B_{i} \in f}\left[r\left(l, B_{i}\right) \times\left|B_{i}\right|\right]
$$

\section{Effective Utilization on Radio Channel Resource}

In this section we further analyze how to utilize the available channel resource to meet the desired performance objectives over BWA communication networks. Note that traffic sessions in BWA access system can be divided into two classes: one is the QoS session[4][5] such as UGS with specific requirement on bandwidth and delay; the other is the Non-QoS session (i.e., BE) without any QoS requirement. All the $\mathrm{BE}$ sessions will contend for the available bandwidth in a best effort manner. Here we propose an effective channel usage (ECU) scheme to optimize the channel utility.

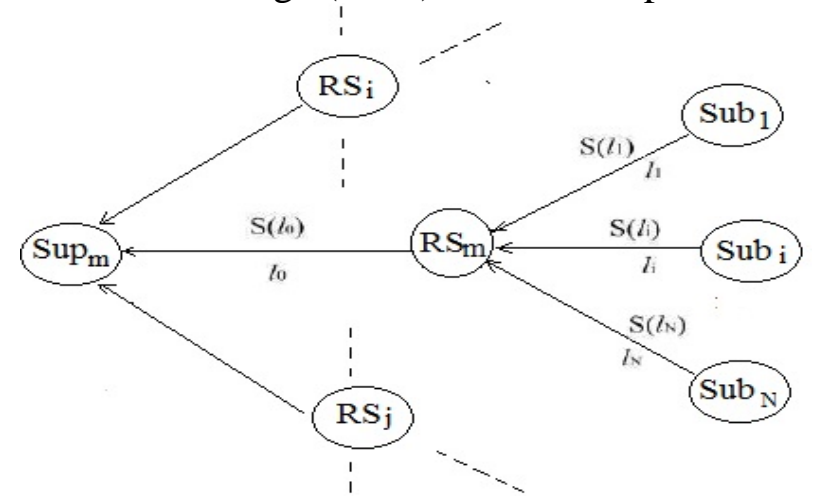

Fig. 2. Channel Transmission Model

Figure 2 illustrates part of a transmission model for the $802.16 \mathrm{~m}$ network, where station $R S_{\mathrm{m}}$ represents a generic relay station (RS) within the network. Stations $S u b_{1}, S u b_{1}, \ldots, S u b_{N}$ are the $N$ 
subordinate stations of $R S_{\mathrm{m}}$, while $\operatorname{Sup}_{\mathrm{m}}$ is its superordinate station. Links $l_{1}, l_{\mathrm{i}}, \ldots, l_{\mathrm{N}}$ are the relevant subordinate links and link $l_{0}$ is the superordinate link. Suppose the total available resource (i.e., time slots or bursts) allocated to the transmission model (regarding $R S_{\mathrm{m}}$ ) in Fig. 2 is denoted by $S(m)$. Specifically we can define a utility function, say, $U(S(m))$ among the competing links as

$$
U(S(m))=\sum_{i=0,1, \ldots, N}\left[\sum_{B_{k} \in S\left(l_{i}\right)} r\left(l_{i}, B_{k}\right) \times\left|B_{k}\right|\right]
$$

where $r\left(l_{\mathrm{i}}, B_{\mathrm{k}}\right)(i=0,1,2, \ldots, N)$ denotes the normalized channel transmission rate over link $l_{\mathrm{i}}$ with respect to burst $B_{\mathrm{k}}$. Parameter $S\left(l_{\mathrm{i}}\right)$ denote the subset of time slots (bursts) allocated to link $l_{\mathrm{i}}$. Hence the utility function in Eq. (3) represents the total transmission throughput produced at the transmission model regarding resource $S(m)$. Our objective is to maximize the usage of channel resource by achieving both the QoS performance satisfaction and optimized system throughput. Accordingly we formulate the following optimization problem as

$$
\begin{array}{cl}
\begin{array}{c}
\text { Maximize } \\
\text { Subject to }
\end{array} & U(S(m)) \\
& X(i)=R(i) \\
& W(i) \leq W_{\max }(i)
\end{array}
$$

Formulation (4) aims to maximize system transmission throughput with respect to QoS performance constrains of (5), (6). Specifically the transmission rate $X(i)$ allocated to a QoS flow $i$ should meet its rate demand $R(i)$ without contention. While the waiting time $W(i)$ for QoS flow $i$ should not exceed its maximal tolerant delay (deadline) $W_{\max }(i)$. Due to different performance requirements, we deal with QoS and Non-QoS traffic via different strategies: As for those QoS traffic flows, we handle them in a priority manner so that the one(s) with the most urgent delay demand are chosen for transmission at each schedule round. As for those Non-QoS traffic flows, we handle them in an opportunistic manner to achieve a maximal system traffic throughput.

Aside from the above effective channel usage(ECU) scheme, if we only focus on maximizing the system traffic throughput, then each available resource (burst) will be allocated in a greedy manner among different links, so that the link with the highest channel transmission rate will be preferred at each schedule round. We call this scheme as the greedy channel usage(GCU) scheme, which be used to make a comparison with the ECU scheme in the experiment part.

\section{Performance Analysis on Channel Utilization Scheme}

In this section we further present an analytical model to analyze the relevant implementation performance metrics for the proposed utilization scheme. Specifically we employ the network calculus theory[7] method, which is based on min-plus algebra theory and focuses on the analysis of performance bounds using the cumulative amount of traffic arrivals or services.

Traffic Arrival Curves

Let's assume that the arrivals to and departures from an arbitrary RS node, say $R S_{\mathrm{m}}$ as in Fig. 2 within time interval $[0, \mathrm{t}]$ are denoted by $\mathrm{A}^{\mathrm{I}}(\mathrm{t})$ and $A^{\mathrm{O}}(t)$ respectively. According to the network calculus theory, the arrival curve of a traffic flow is bounded by the its arrival envelope which is regulated by the peak traffic rate, say $r\left(l_{i^{*}}\right)$ (i.e., $\max \left(r\left(l_{\mathrm{i}}\right)\right)$ and its arrival curve is determined as

$$
A^{I}(t) \leq \inf _{0 \leq s \leq t}\left\{A^{I}(s)+r\left(l_{i^{*}}\right)(t-s)\right\}
$$

\section{Traffic Departure Curves}

The departure curve is related with both the arrival and service curves. Specifically, the service curve is determined by the output link bandwidth $r\left(l_{0}\right)$ as described above, and the departure curve of the traffics out of $R S_{\mathrm{m}}$ is bounded by

$$
A^{O}(t) \geq \inf _{0 \leq s \leq t}\left\{A^{I}(t)+r\left(l_{0}\right)(t-s)\right\}
$$




\section{Buffer Occupancy}

During each time interval [0, t], the backlog at $R S_{\mathrm{m}}$ is related to both the incoming traffics into it and the outgoing traffic out of it. Under the network calculus model, the backlog can be determined as the vertical difference between the arrival and departure curves. Accordingly, the mathematical formula of the buffer occupancy at $R S_{\mathrm{m}}$ is defined as

$$
Q_{m}(t)=A^{I}(t)-A^{O}(t)
$$

While the maximal buffer occupancy at \$RS_m\$ is bounded by

$$
Q_{m}(\max ) \leq \sup _{s \geq 0}\left\{A^{I}(\max )-r\left(l_{0}\right)(s)\right\}
$$

\section{Traffic Delay}

Under the network calculus, the traffic delay is determined as the horizontal difference between the arrival curves and departure curves. Accordingly the traffic delay at $R S_{\mathrm{m}}$ is defined as

$$
W_{m}(t)=\inf \left\{w \geq 0: A^{I}(t)-A^{O}(t+w) \leq 0\right\}
$$

\section{Traffic Throughput}

This performance measure indicates the average rate of traffics successfully transmitted by $R S_{\mathrm{m}}$ within a given time interval. Specifically the loss ratio of the traffics at $R S_{\mathrm{m}}$ is calculated as $P_{\mathrm{m}}(\mathrm{t})=\left[\max \left\{0, Q_{\mathrm{m}}(\mathrm{t})-Q_{\mathrm{m}}\right\}+A^{\exp }(t)\right] / A^{\mathrm{I}}(t)$, where $A^{\exp }(t)$ denotes the amount of traffics whose delay expires the deadline requirements. Based on these, the average effective traffic throughput at $R S_{\mathrm{m}}$ within a certain time interval $[0, t]$ is evaluated as

$$
T R_{m}(t)=\left(1-P_{m}(t)\right) A^{I}(t)
$$

\section{Conclusion}

This paper aims to exploit the optimization on radio channel resources in $802.16 \mathrm{~m}$ networks to meet the user application requirements. To conquer the challenges caused by the state variation of communication channels, we designed the novel schemes to specify the channel state and exploit the maximal channel transmission capacity. Moreover, an effective channel usage scheme is proposed to fully utilize the channel bandwidth regarding the user actual demand. We also make the performance analysis with the adoption of network calculus for the proposed schemes. The simulation results validate that the our scheme can achieve both QoS satisfactory and throughput optimization.

\section{Acknowledgements}

This work is fully supported by National Natural Science Foundation (NSF) of China with grant No.61202461, and National Natural Science Foundation (NSF) of China with grant No. 61472129.

\section{References}

[1] IEEE 802.16m-08/004r3, IEEE 802.16m Evaluation Methodology Document (EMD), IEEE, Oct. 2008.

[2] IEEE P802.16m/D6, Draft Amendment to IEEE Standard for Local and Metropolitan Area Networks Part

16: Air Interface for Fixed and Mobile Broadband Wireless Access Systems, IEEE, May 2010.

[3] IEEE 802.16m-08/003r7, IEEE 802.16m System Description Document (SDD), IEEE, Feb. 2009.

[4] Jeffrey G. Andrews and Arunabha Ghosh and Rias Muhamed, Fundamentals of WiMAX: understanding broadband wireless networking, Upper Saddle River, NJ: Prentice Hall,2007.

[5] IEEE Standards, IEEE Draft Standard for local and metropolitan area networks Part 16: Air Interface for Broadband Wireless Access Systems, pp. 1-2614, March 2012.

[6] M. -S. Alouini and A. J. Goldsmith, Adaptive modulation over Nakagami fading channels, Kluwer Journal on Wireless Communication, vol. 13, no. 1-2, pp. 119-143, 2000.

[7] C.Lee, A. Burchard, and J. Liebeherr, A network calculus with effective bandwidth, IEEE/ACM Transactions on Networking(TON), vo.15, no.6, pp.1442-1453, December 2007. 